\title{
Resonant ultrasound spectroscopy for measurement of mechanical damping: Comparison with broadband viscoelastic spectroscopy
}

\author{
T. Lee \\ Department of Biomedical Engineering and Engineering Mechanics Program, University of Wisconsin- \\ Madison, 147 Engineering Research Building, 1500 Engineering Drive, Madison, Wisconsin 53706-1687
}

R. S. Lakes ${ }^{\text {a) }}$

Department of Biomedical Engineering, Department of Engineering Physics, Engineering Mechanics Program, Materials Science Program, and Rheology Research Center, University of Madison-Wisconsin, 147 Engineering Research Building, 1500 Engineering Drive, Madison, Wisconsin 53706-1687

A. Lal

Department of Biomedical Engineering and Department of Electrical and Computer Engineering, University of Wisconsin-Madison, 2438 Engineering Hall, 1415 Engineering Drive, Madison, Wisconsin 53706-1687

(Received 18 February 2000; accepted for publication 14 March 2000)

\begin{abstract}
This article compares resonant ultrasound spectroscopy (RUS) and other resonant methods for the determination of viscoelastic properties such as damping. RUS scans from 50 to $500 \mathrm{kHz}$ were conducted on cubical specimens of several materials including brass, aluminum alloys, and polymethyl (methacrylate) (PMMA), a glassy polymer. Comparison of damping over the frequency ranges for broadband viscoelastic spectroscopy (BVS) and RUS for indium tin alloy in shear modes of deformation discloses a continuation of the $\tan \delta$ power-law trend for ultrasonic frequencies up to $300 \mathrm{kHz}$. For PMMA, resonant peaks were sufficiently broad that higher modes in RUS began to overlap. Tan $\delta$ via RUS and BVS for PMMA agreed well in the frequency range where the methods overlap. RUS is capable of measuring $\tan \delta$ as high as several percent at the fundamental frequency. Since higher modes are closely spaced, it is impractical to determine $\tan \delta$ above $0.01-0.02$ at frequencies other than the fundamental. () 2000 American Institute of Physics.
\end{abstract}

[S0034-6748(00)01907-9]

\section{INTRODUCTION}

Resonant ultrasound spectroscopy (RUS) involves scanning the resonance structure of a compact specimen such as a cube, parallelpiped, or short cylinder. ${ }^{1-3}$ In a typical embodiment of the method, a rectangular parallelpiped or cubic sample is supported by transducers at opposite corners. Corners are used since they always move during vibration (they are never nodes) and they provide elastically weak coupling to the transducers. Piezoelectric ceramics such as leadzirconate-titanate (PZT) plates are suitable as broadband transducers. One transducer excites the vibration, and the other converts the resonant motion to an electrical signal. The specimen is supported by contact pressure; it does not require cementing or the use of coupling agents. The resonance structure, from about $50 \mathrm{kHz}$ to more than $10 \mathrm{MHz}$ depending on the specimen size, is complex, however advances in computation capacity have enabled the back calculation of material properties from the resonance frequencies. RUS is capable of determining all the elastic moduli $C_{i j k l}$ of one specimen of an anisotropic material. The method is not capable of static or low frequency studies. The use of mechanical resonance to infer material properties is not new; it may predate the industrial revolution. ${ }^{4}$ British railroad engi-

\footnotetext{
a) Author to whom correspondence should be addressed; electronic mail: lakes@engr.wisc.edu
}

neers tapped the wheels of a train and used the sound to detect cracks. ${ }^{4}$ Mechanical resonance of tissue has been used as a diagnostic tool for hundreds of years. Physicians are able to obtain important diagnostic information by pressing the patient's body with one finger and tapping (percussing) with another finger. The sounds produced and the tactile sensations felt reveal to the examiner the condition of the underlying tissue. ${ }^{5,6}$ Percussion as a diagnostic tool dates from a publication in 1761 by Auenbrugger. ${ }^{7}$ Percussion sounds have also been used ${ }^{8}$ to diagnose bone fracture healing.

Some studies of damping have been done via RUS, e.g., study of $\mathrm{Ni}_{80} \mathrm{P}_{20}$ has disclosed $\tan \delta$ in the range $10^{-4}-2$ $\times 10^{-3}$ at $320 \mathrm{kHz}$ over a range of temperature. ${ }^{9}$ Here $\delta$ is the phase angle between stress and strain, or the ratio of imaginary to real part of the modulus. Tan $\delta$ is also the inverse of the quality factor $Q$. Mechanical damping is important in applications of vibration abatement and as a probe of phase transitions, defects in crystals, and dislocation mobility. In principle, ${ }^{3}$ one can determine the full anisotropy of mechanical $Q$, however the method has not been developed. One difficulty is the identification of the vibration mode in question. Damping, $\tan \delta$, can be determined but interpretation depends on identification of the vibration mode. Most resonant frequencies in RUS depend on combinations of elastic constants, ${ }^{3}$ and the width of resonant peaks depends on combinations of complex moduli. ${ }^{10}$ Broadband viscoelastic spectroscopy $(\mathrm{BVS})^{11}$ covers a wider range of frequency, 
11 decades (a factor of $10^{11}$ ) of time and frequency at subresonant frequencies and at pure resonant modes. BVS is restricted to damping in torsion (shear) and bending (axial deformation). The wide range of time and frequency was achieved by minimizing the inertia attached to the specimen, using a simple specimen geometry governed by a known analytical solution amenable to numerical inversion, and using driving and detection methods free of resonances and also capable of quasistatic tests.

In this article, we explore the utility of RUS for mechanical damping measurements, particularly for materials of high damping.

\section{THEORY}

\section{A. Resonance of an isotropic cube}

Most of the natural frequencies $\nu$ of a vibrating cube, even an isotropic one, are not amenable to solution by analytical means. However the Mindlin-Lamé ${ }^{12}$ modes have an analytical solution

$$
\nu=\frac{m}{L \sqrt{2}} \sqrt{\frac{G}{\rho}},
$$

with $\mu=G$ as the shear modulus, ${ }^{13} \rho$ as density, $L$ as the length of a cube side, and $m$ as an integer. This formulation can handle anisotropy. For example, $G$ is replaced by the modulus element $C_{44}, C_{66}$, or $C_{55}$ depending on sample orientation. For an isotropic material, the principal shear moduli are equal: $C_{44}=C_{66}=C_{55}=G$.

Following Demarest ${ }^{14}$ the lowest mode is a torsional mode

$$
\nu=\frac{\sqrt{2}}{\pi L} \sqrt{\frac{G}{\rho}} .
$$

The torsion frequency is lower than Mindlin-Lamé by a factor of about 1.57. Demarest used numerical methods to determine the natural frequencies of the remaining modes which are shown in Fig. 1. Mindlin ${ }^{15}$ analytically derived the torsion modes of long elastic bars, however a short bar or cube differs from a long bar, since warp motion is more free in the case of a cube.

\section{B. Resonance of a circular cylinder}

For the torsional oscillation of a circular cylindrical rod of a linearly viscoelastic material, fixed at one end and free of constraint, with an applied moment (sinusoidal in time) at the other end, the stiffness and inertia are distributed throughout the entire rod as in cube resonance. This rod resonance configuration is referred to as "fixed-free" in the experimental literature, and it is the configuration used in the BVS system. The dynamic structural rigidity ${ }^{16}$ is the ratio of applied external moment $M$ to the angular displacement $\theta$; the rigidity is complex since $M$ and $\theta$ need not be in phase. In the following the compliance, which is the inverse of the rigidity, is normalized to its quasistatic value

$$
\Gamma=\left|\left[\left(\frac{1}{2} \rho \pi R^{4}\right)\left(\omega^{2} L\right) \frac{\cot \Omega^{*}}{\Omega^{*}}-I_{\mathrm{at}} \omega^{2}\right]^{-1}\right|\left|\frac{\pi R^{4}}{2 L} G^{*}\right|,
$$

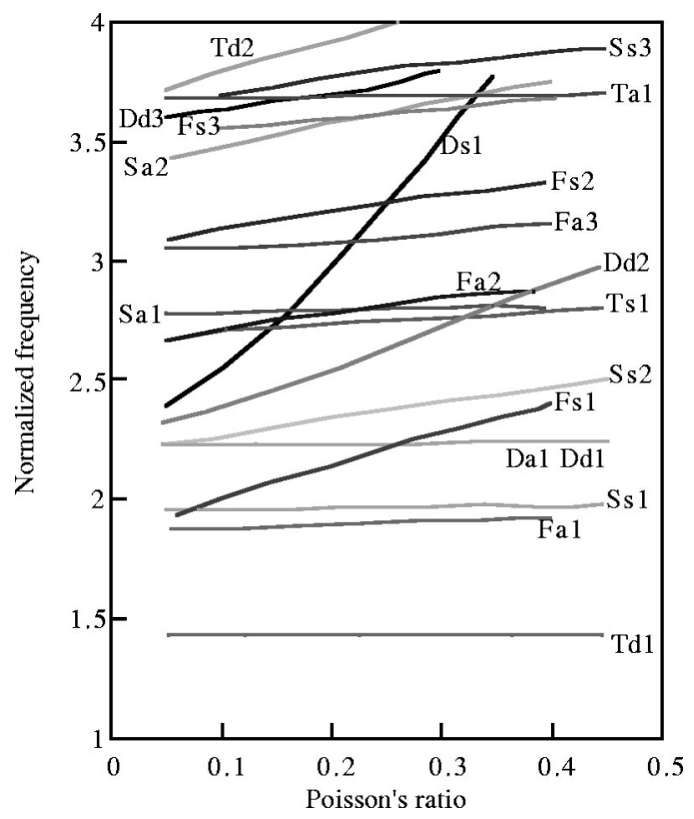

FIG. 1. Normalized frequencies of an isotropic cube as a function of Poisson's ratio (adapted from Ref. 14).

in which

$$
\Omega^{*}=\sqrt{\frac{\rho \omega^{2} L^{2}}{G^{*}}},
$$

with $I_{\text {at }}$ as the attached mass moment of inertia, $L$ as the rod length, and $R$ as the radius of the rod. The loss tangent $\tan \delta$ is defined as the ratio of the imaginary to the real part of $G^{*}$. The lowest natural frequency is, for fixed-free torsional vibration in the absence of end inertia,

$$
\nu=\frac{1}{4 L} \sqrt{\frac{\left|G^{*}\right|}{\rho}} .
$$

Higher modes occur in the ratio $1,3,5,7,9 \cdots$ in the absence of damping. The lowest natural frequency for a cylinder with both ends free ("free-free" configuration) is twice as large as in Eq. (5), and about $10 \%$ greater than for a cube of the same length following Eq. (2). One can study resonance of a free-free cylinder via RUS, but many other modes are present in addition to torsional ones.

\section{Determination of damping $\tan \delta$}

Mechanical damping $\tan \delta$ can be extracted from experimental data by fitting an exact analytical expression such as Eq. (3) to the cylinder resonance data. Such a procedure cannot be done for RUS since there is no exact analytical expression for the cube resonance structure. For small $\tan \delta$, one may use several approximation schemes to infer damping from a peak of the resonance curve. For example, in the half-width approach, the dynamic compliance is determined at a resonance; then the oscillator is tuned until the response is half the value at resonance. The damping is given by

$$
\tan \delta \approx \frac{1}{\sqrt{3}} \frac{\Delta \omega}{\omega_{1}},
$$


TABLE I. Specimen dimensions.

\begin{tabular}{llcc}
\hline \hline \multicolumn{1}{c}{ Material } & \multicolumn{1}{c}{ Dimension $(\mathrm{cm})$} & Tolerance $(\mathrm{cm})$ & Mass $(\mathrm{g})$ \\
\hline Brass & $1.001 \times 1.001 \times 1.001$ & \pm 0.001 & 8.175 \\
A $1(6061-T 651)$ & $1.001 \times 1.002 \times 0.998$ & \pm 0.002 & 2.682 \\
A $1(6061-T 6)$ & $0.44 \times 0.44 \times 0.44$ & \pm 0.001 & 0.229 \\
InSn & $0.228 \times 0.230 \times 0.230$ & \pm 0.002 & 0.085 \\
PMMA & $0.250 \times 0.258 \times 3.070$ & \pm 0.002 & 0.218 \\
PMMA & $1.04 \times 1.04 \times 1.02$ & \pm 0.001 & 1.196 \\
\hline \hline
\end{tabular}

in which $\omega_{1}$ is the angular frequency at a resonance. The $\Delta \omega$ represents the full width of the resonance curve at half maximum amplitude. The relative width of the resonance curve is referred to as $Q^{-1}$, with $Q$ as the "quality factor," a term also used in describing resonant behavior in other systems including electric circuits. Derivation of exact relations between $Q$ and $\tan \delta$ for high values of damping generally requires a model of the viscoelastic response of the material. ${ }^{17}$ In rubbery materials for which $\tan \delta$ approaches 1 , numerical techniques based on Newton's method were used to extract material phase angles from structural phase angles near resonances ${ }^{18}$ of a cylinder following Eq. (3).

One may also fit the data to a Lorentzian function as follows. For a lumped system in which the attached inertia is large, the normalized dynamic torsional compliance is ${ }^{19}$

$$
\Gamma=\sqrt{\frac{1}{\left[1-\left(\nu / \nu_{0}\right)^{2}\right]^{2}+\tan ^{2} \delta}},
$$

in which $\tan \delta$ is the loss tangent, a measure of mechanical damping. The dependence of this $\Gamma$ on frequency is referred to as a Lorentzian function, sometimes called a Lorentzian line shape in view of the form of the spectral lines associated with electromagnetic radiation (light) from atomic resonances. The Lorentzian is a reasonable approximation of the resonant peak of a distributed system as well, provided the damping is small.

\section{PROCEDURES}

\section{A. Materials}

Several solid materials including brass, polymethyl (methacrylate) (PMMA), and aluminum alloy (6061-T6, 6061-T651) were used for this research as shown in Table I. Each specimen was cut using a low-speed diamond saw. The cubic brass sample is composed of $89 \%$ copper, $5 \%$ tin, and $3 \%$ zinc and is commonly known as "Lead-Free 836", (FEDERALLOY I-836®, Southern Alloy of America, Inc., P.O. Box 1150, Salisbury, NC 28145). The material properties, Young's modulus $E=106.9 \mathrm{GPa}$ and Poisson's ratio 0.35 were provided by the manufacturer.

PMMA, a viscoelastic polymer also called Plexiglas ${ }^{\circledR}$, has a loss tangent of about 0.1 at $1 \mathrm{~Hz}$ and decreases to around 0.03 near $1 \mathrm{kHz} .{ }^{20}$ PMMA has a Young's modulus of $3.5 \mathrm{GPa}$ and a shear modulus of $1 \mathrm{GPa}$ near $1 \mathrm{~Hz} .{ }^{20} \mathrm{~A}$ specimen of PMMA was prepared with dimensions shown in Table I. The tolerance on the specimen varied according to the difficulty of machining the specimen.

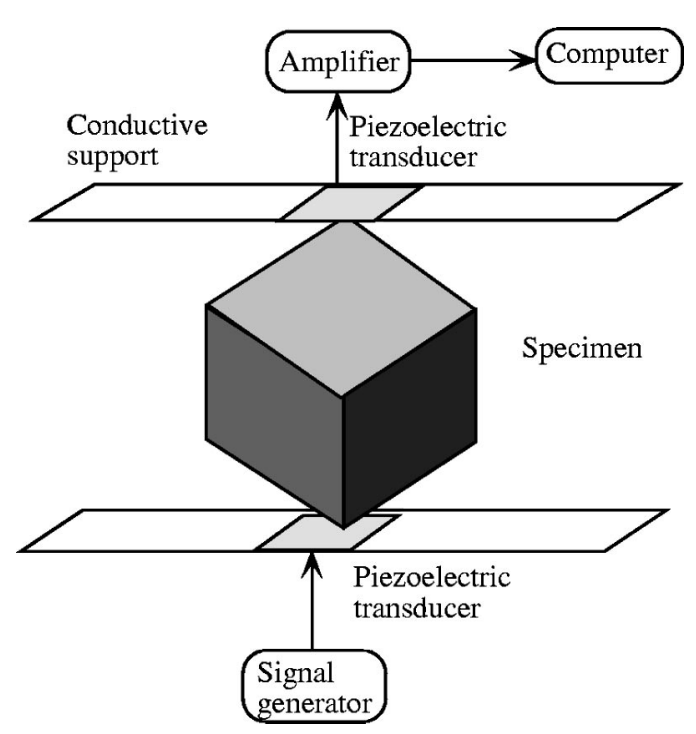

FIG. 2. Schematic diagram of RUS apparatus.

Several aluminum alloy specimens were studied. These were type 6061-T6, for which ${ }^{21} E=68.9 \mathrm{GPa}, \quad G$ $=25.9 \mathrm{GPa}, \tan \delta \approx 3.6 \times 10^{-6}$, and type 6061-T651 for which $E=69 \mathrm{GPa}, G=26 \mathrm{GPa}$.

\section{B. RUS procedure}

The RUS system is made by Dynamic Resonance Systems, (255 Lane 13, Powell, WY 82435), model DRS modulus I. It consists of two data processing boards and one set of PZT transducers. Each transducer has a face diameter of 4 $\mathrm{mm}$ and length of $3 \mathrm{~mm}$. Each specimen was mounted by its corners between the transducers, as shown in Fig. 2. The PZT probes were offset from the center of the sample in the mounting process. The transmitting transducer was connected to the rf output transducer, which drives the specimen. The other transducer converts the resonant motion of the specimen to an electrical signal. The transducers are mounted on symmetrically mounted plastic arms. Each arm is supported by two springs so that one can adjust the height of the transducers. For isotropic materials, there was no need to keep track of sample direction during mounting, while the anisotropic samples were placed with careful reference to their anisotropic axis. Data processing was done using software developed by DRS Inc in an IBM Pentium PC connected to the RUS system.

By using Eq. (2), the lowest torsional mode $\nu$ was calculated to set the frequency range to be scanned. Transmitting transducer gain was suitably set to achieve a clear spectrum. Given settings for the frequency range and the number of data points, the RUS system plotted the resonant response spectrum. The real and imaginary parts of the signal were saved and exported. Data were plotted using Kaleidagraph graphic software and a Lorentzian function was fitted to selected resonant peaks for inference of damping.

\section{BVS procedure}

Viscoelastic testing was performed using the modified $^{11,22}$ broadband viscoelastic spectroscopy 


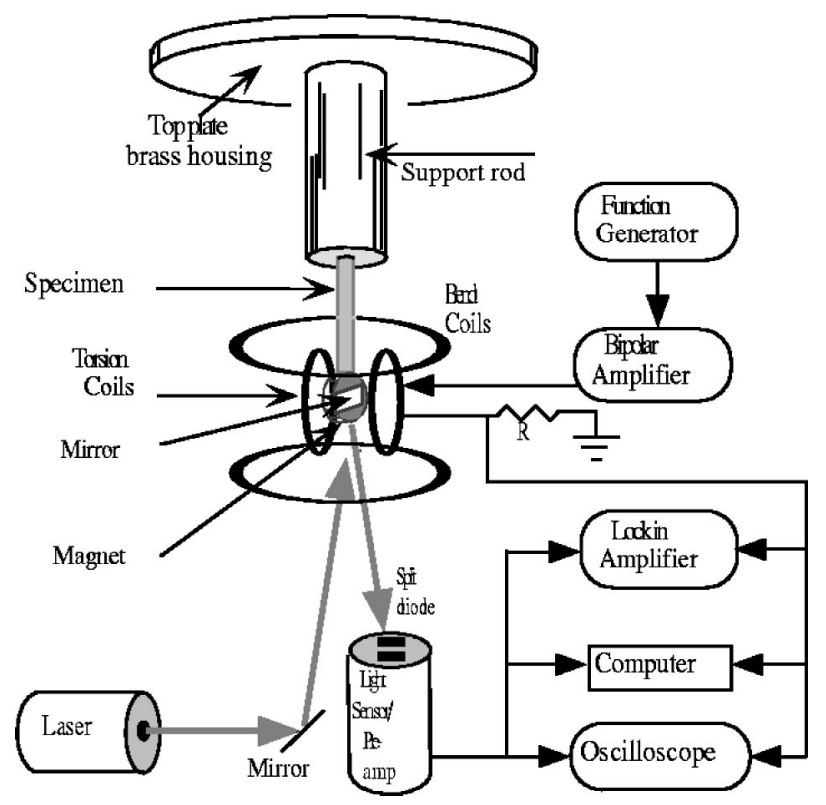

FIG. 3. Schematic diagram of BVS apparatus.

apparatus $^{18}$ shown in Fig. 3. The instrument is capable of torsion and bending studies in creep, subresonant dynamic and resonant dynamic oscillation upon the same specimen. The wide frequency range capability of this apparatus is particularly useful in testing composites and other materials which are thermorheologically complex. The specimen was mounted using a cyanoacrylate cement at the top to its support rod and at the bottom to a high intensity neodymium iron boron magnet. The magnet used here had dimensions $2.72 \times 2.92 \times 3.34 \mathrm{~mm}$ and a mass of $0.185 \mathrm{~g}$. The mirror mass was $0.012 \mathrm{~g}$. Two mirrors were used since it was found that the asymmetrical inertia of a single mirror on the magnet caused undesirable coupling between torsion and bending.

Dynamic experiments were conducted by applying an amplified sinusoidal voltage from a digital function generator to the Helmholtz coil. This coil imposed a magnetic field on the permanent magnet and transmitted an axial torque on the specimen. The angular displacement of the specimen was measured using laser light reflected from a mirror mounted on the magnet to a split-diode light detector. The detector signal was amplified with a wideband differential amplifier. Torque was inferred from the Helmholtz coil current. Torque calculations were supported by calibrations using the wellcharacterized type $6061 \mathrm{Al}$ alloy described above. ${ }^{18}$ Input and output voltages were recorded using a digital data acquisition system containing a Macintosh computer and LabVIEW® interface hardware and software, which was also used to determine the phase angle at the lower frequencies. The phase angle was determined using a lock-in amplifier (SRS 850) with claimed phase resolution $0.001^{\circ}$. The frequency range was segmented into two regions since the wide frequency range necessitated different time constant settings.

A Lorentzian curve fit was used to obtain mechanical damping for each specimen. A theoretical curve for the resonant regime of PMMA was generated using Eq. (3). Input data were the shear modulus at $1 \mathrm{kHz}$, the specimen dimensions and mass, and the end inertia $\left(4.33 \times 10^{-10} \mathrm{~kg} \mathrm{~m}^{4}\right)$ cal-

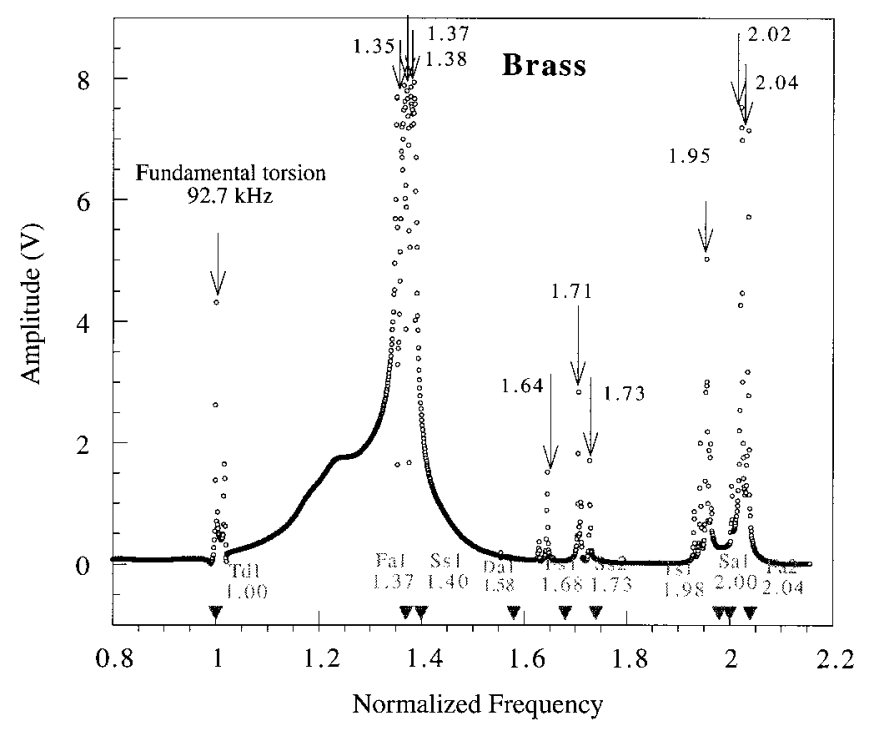

FIG. 4. RUS spectrum for a brass cube. Received amplitude in volts vs frequency. Theoretical modes (Demarest) for a perfect isotropic cube of Poisson's ratio of 0.35 are shown at bottom for comparison. Numbers above peaks indicate normalized frequency.

culated from the mass and dimensions of the magnet and mirrors. Damping was assumed to follow a power law over the limited frequency range of the resonances, with the slope determined from damping inferred from Lorentzian fits of the two lowest modes.

\section{RESULTS AND DISCUSSION}

There are no standard materials for viscoelasticity studies, since the physical processes which give rise to viscoelastic behavior tend to depend on specimen preparation methods as well as on environmental variables such as temperature and humidity. Therefore detailed quantitative comparisons with other results cannot be made. Even so, one may make comparisons between RUS and BVS for the same specimen as well as with the literature.

A representative RUS spectrum for a brass cube is shown in Fig. 4. The experimental frequency scale was normalized to the first torsional mode at $92.7 \mathrm{kHz}$. The triangles on the horizontal axis of the graph show the theoretical normal mode frequencies calculated following Demarest. ${ }^{14}$ The experimental torsional (lowest) mode was split into two peaks differing by about $1.6 \%$. The splitting cannot be ascribed to deviations from perfect cubic shape since these deviations would only account for $0.1 \%$ splitting (see Table I). The splitting is therefore due to anisotropy. The lowest mode is clearly distinguishable because the torsional frequency is far from the others as in Fig. 1. Higher modes are closer together; even so, the peaks are well defined since they are sharp. Experimental resonant frequencies generally agree with the theoretical resonant frequencies from Demarest. ${ }^{14}$ An expanded plot of the lowest mode is shown in Fig. 5. The inferred tan $\delta$ for the brass cube calculated by using the Lorentzian curve fit, Eq. (7), for the lowest (torsional) mode is $\tan \delta=4.4 \times 10^{-4}$. The half width approach, Eq. (6), gave $\tan \delta=5 \times 10^{-4}$ to one significant figure owing 


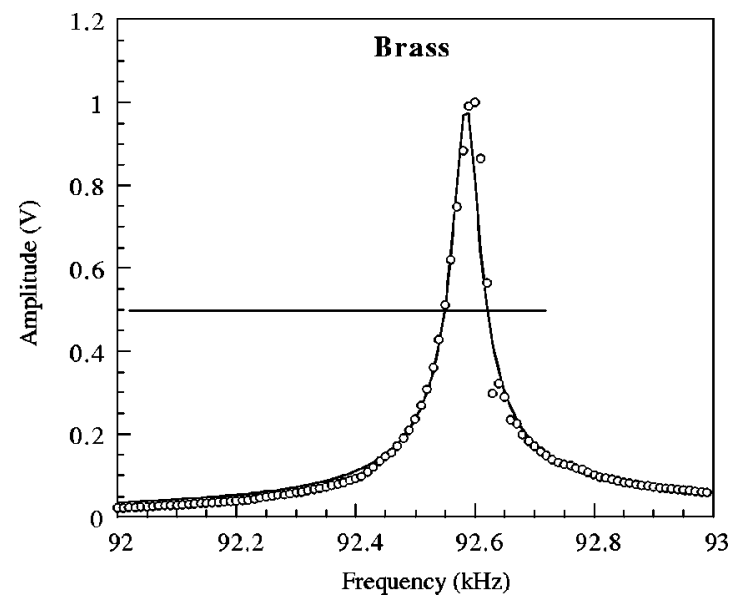

FIG. 5. Detail of RUS spectrum of a brass cube, with curve fit of a Lorentzian (solid line).

to the sparse set of data points in the narrow peak. Brass is known to have a low damping, less than 0.001 , in harmony with the present results.

The RUS spectrum of aluminum alloy 6061-T651 is shown in Fig. 6. The aluminum sample was smoother than brass making it more difficult to mount and align the cube in the RUS system. The experimental frequency was normalized at $142.9 \mathrm{kHz}$ where the first mode was observed. The experimental torsional (lowest) mode was split into two peaks differing by about $2 \%$. The splitting cannot be ascribed to deviations from perfect cubic shape since these deviations would only account for $0.2 \%$ splitting (see Table I). The splitting is therefore due to anisotropy, possibly due to rolling during the manufacturing process. Inferred $\tan \delta$ was $8.8 \times 10^{-4}$ at $144 \mathrm{kHz}$. For aluminum alloy 6061-T6, inferred $\tan \delta$ was $2.2 \times 10^{-4}$ at $331 \mathrm{kHz}$. The true damping is much lower: $\tan \delta=3.6 \times 10^{-6}$, measured at $1 \mathrm{kHz}$ in a low-background lumped resonator. ${ }^{21}$ Therefore the apparent damping is dominated by radiation of ultrasound into the air.

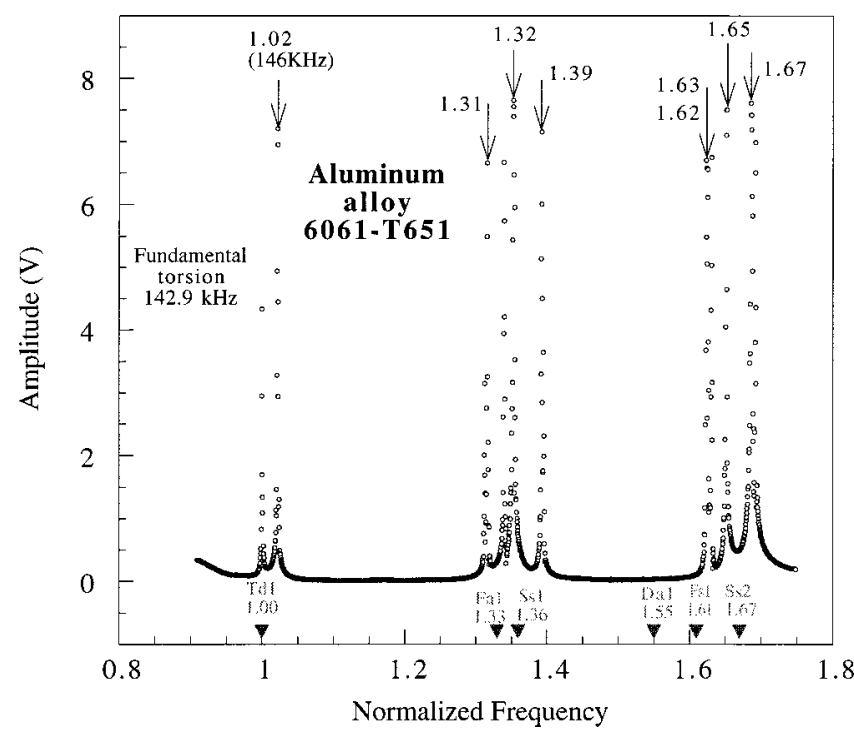

FIG. 6. RUS spectrum for an aluminum alloy cube, type T651. Theoretical modes (Demarest) for a perfect isotropic cube of Poisson's ratio of 0.3 are shown for comparison.

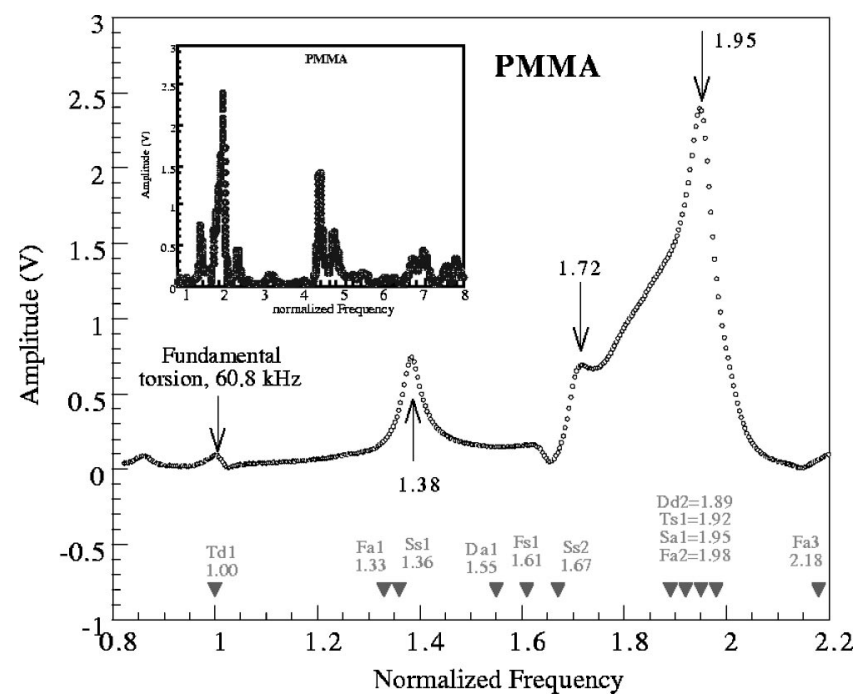

FIG. 7. RUS spectrum for a PMMA cube. Theoretical modes (Demarest) for a perfect isotropic cube of Poisson's ratio of 0.35 are shown at bottom for comparison. (Inset) spectrum over a wider range of frequency.

This is not an intrinsic limitation of RUS, as discussed below.

PMMA has a higher mechanical damping $\tan \delta$ than brass or aluminum. The RUS frequency spectrum for a PMMA cube is shown in Fig. 7. The inferred $\tan \delta$ calculated by using the Lorentzian curve fit for the lowest (torsional) mode is 0.032 . Observe that the higher modes for PMMA in the RUS results overlap and are not distinguishable, since the comparatively high $\tan \delta$ of this polymer gives rise to broad resonant peaks. For example, modes $\mathrm{Fal}$ and $\mathrm{Ss} 1$ are the next higher modes after the fundamental for normal values of Poisson's ratio, following Fig. 1. These modes are separated by 0.037 of the Fa1 frequency. Following Eq. (6), if $\tan \delta$ $\sim 0.037$, the mode peaks will overlap, precluding a meaningful measurement. For $\tan \delta=0.02$ one can begin to distinguish the peaks if they are of equal magnitude. If one were to use the method of resonant half widths, Eq. (6), interpretation of a split peak would be problematical unless $\tan \delta$ were less than about 0.005 . It is possible that overlap of higher modes could be eliminated by selectively exciting them as pure modes by control of specimen angulation, but that would complicate the RUS system. The Ds1 mode shown in Fig. 1 would be of particular interest since it reveals viscoelasticity in the bulk modulus, hence aspects of free volume in the case of polymers. However for realistic values of Poisson's ratio it overlaps several other modes.

PMMA dynamic compliance spectra via BVS are plotted in Fig. 8. Both experimental and theoretical data in Fig. 8 are normalized to the value at $1 \mathrm{kHz}$. Also shown is a theoretical curve based on an assumption of magnet end inertia one eighth the actual value. By comparing, one observes the higher modes are reduced in magnitude by the end inertia. In any case, they are more widely separated than in the case of RUS. The mechanical damping inferred from both the subresonant and resonant spectra of PMMA is shown in Fig. 9. These resonances follow the power law curve fit. The damping is presented as a solid line in Fig. 9. Tan $\delta$ from the first torsion mode from RUS results is shown as a solid triangle. 


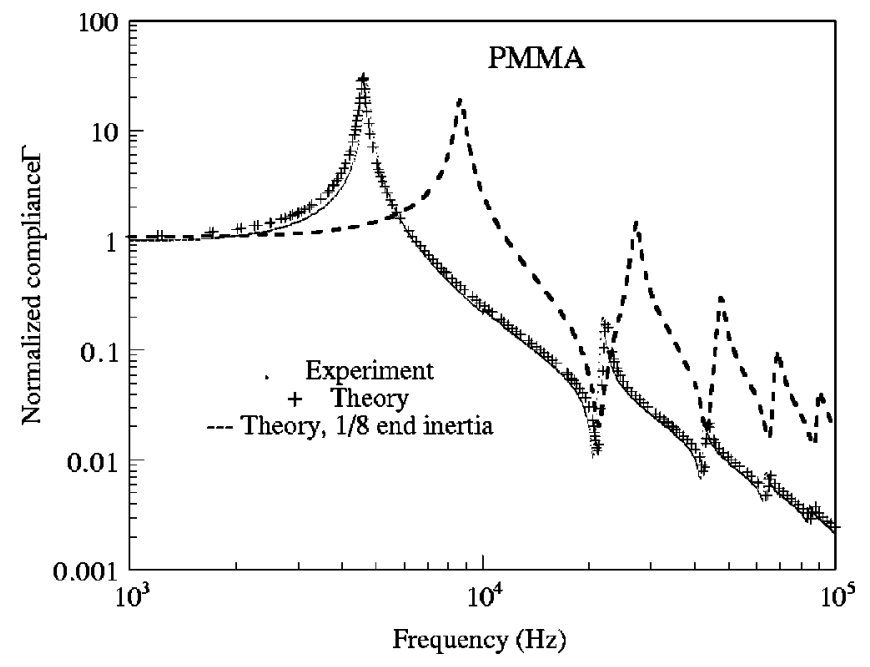

FIG. 8. BVS dynamic compliance spectrum for PMMA: (points) experiment, (crosses, +) theoretical plot based on measured geometrical and inertial properties; (dash line) theoretical plot based on end inertia 1/8 of actual value.

Some of the higher RUS modes follow the power law trend line, which suggests that some of the overlapping modes are excited more strongly than others. The broad damping peak, called a $\beta$ peak, is consistent with the results of Koppellmann. ${ }^{20}$

In existing experimental methods, some modifications are needed if $\tan \delta$ is very large or very small. For low loss materials, it is necessary to eliminate all spurious sources of damping other than that in the specimen itself. ${ }^{23}$ Errors can arise due to radiation of sound energy into the air. These errors cause the apparent loss to be greater than the true material loss. To eliminate such errors, it may be necessary to perform the experiments in an evacuated chamber. For $\tan \delta \leqslant 10^{-4}$, precautions of this type are usually necessary. Sound radiation errors have been studied for RUS. ${ }^{24}$ As one might expect, they are more severe for compressional modes than torsional modes. Moreover, the resonating specimen must be supported in some way, and that support can intro-

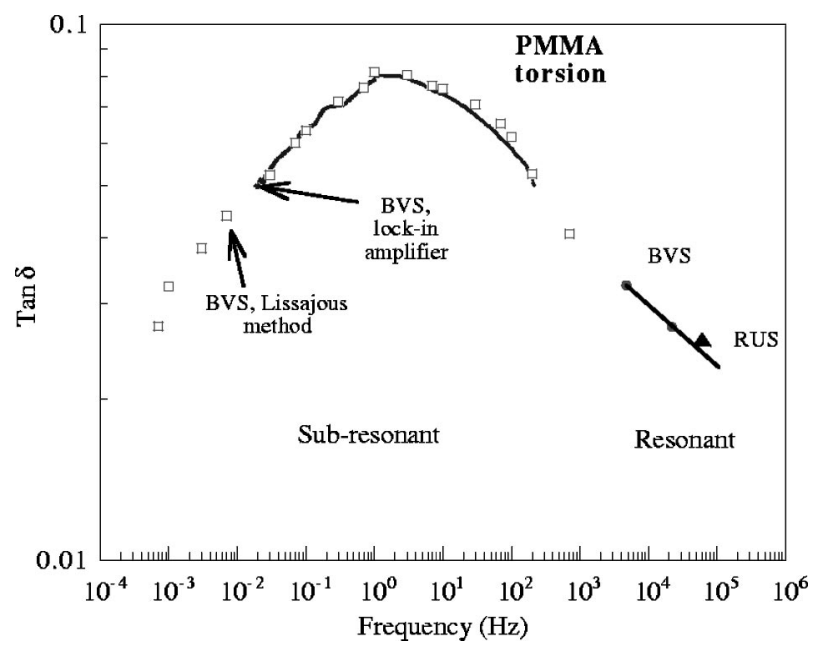

FIG. 9. Tan $\delta$ via BVS and RUS for PMMA: (squares) BVS subresonant Lissajous method, (dense points) BVS lock-in method; (solid circles) BVS lowest two resonances; (solid line) BVS from curve fit in Fig. 8; (solid triangle) RUS lowest mode.

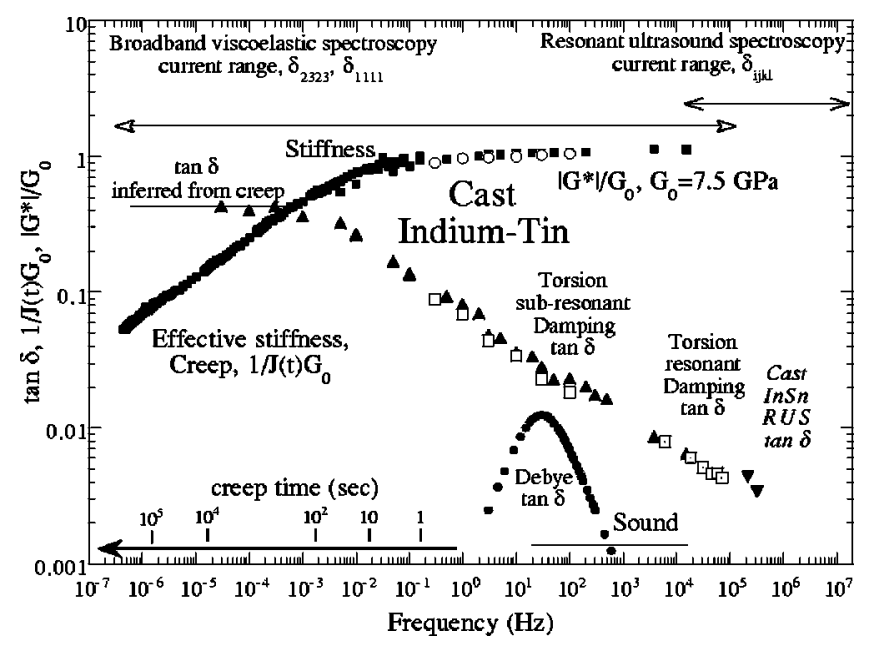

FIG. 10. Comparison of BVS and RUS damping results for cast In-Sn.

duce spurious losses. The point contact used in RUS is advantageous in this regard since it generates a large mismatch in mechanical impedance. Alternatively in other free-free resonance methods, one may support the specimen by a greased thread at a vibration node. ${ }^{23}$ In BVS, parasitic loss due to transmission of waves into the fixed end is minimized by using a comparatively thick support rod of stiff, dense material such as steel or tungsten to achieve a large mismatch in acoustic impedance and by using a slender specimen. For example, ${ }^{19}$ for a metal alloy specimen of diameter $3 \mathrm{~mm}$ and shear modulus $15 \mathrm{GPa}$, the parasitic damping is about $10^{-6}$ below resonance and $5 \times 10^{-4}$ at resonances. The latter value is comparable to air damping. BVS is overall better suited to materials of comparatively high loss, $\tan \delta$ from 0.005 to 1 .

BVS and RUS may be considered complementary methods. For example, by combining BVS and RUS we are able to expand the frequency range of testing. This benefit is clear in Fig. 10 which shows the combined results for cast In-Sn. The capability of measuring mechanical damping was extended by using the RUS system. We remark that cutting and other handling of such a material can alter the dislocation structure, hence the damping.

RUS has the advantage that specimens are held in the apparatus by point contact; there is no need to cement or align anything. RUS allows one to obtain the elements of the elastic modulus tensor from a single specimen. In principle, ${ }^{3}$ one can also determine the full anisotropy of mechanical damping, however the method has not been developed. One difficulty is the identification of the vibration mode in question. A further difficulty is the fact that for viscoelastic materials, we may have an asymmetric modulus tensor $C_{i j k l}^{*}(\nu) \neq C_{k l i j}^{*}(\nu)$ since there is no strain energy function for viscoelastic materials. This can cause a further splitting of peaks in addition to splitting due to imperfect cubes and to anisotropy. This has not been addressed for RUS; the software, which assumes elasticity rather than viscoelasticity, cannot handle such a situation. A further software-related issue is the fact that inversion algorithms used to extract the moduli assume elastic behavior, specifically that moduli are independent of frequency. In viscoelastic materials, damping 
implies dependence of the moduli on frequency $\mathrm{v}$ via the Kramers-Kronig relations. For example, a constant damping $\delta$ entails a modulus which goes as $\mathrm{v}^{2 \delta / \pi}$, so for $\delta=0.01$, a factor of ten increase in frequency increases the modulus by $1.48 \%$. Finally, RUS can only reveal damping of the lowest (torsional) mode in a high-damping material such as a polymer, as we have observed in this study. The reason is that high damping means broad peaks. Higher modes in RUS are closely spaced, and broad peaks will overlap.

BVS has the advantage of a very wide frequency range with no need to appeal to time-temperature superposition. It is therefore particularly suited to composite materials and biological materials. Anisotropy can be studied, albeit by preparing a set of specimens in different directions.

\section{ACKNOWLEDGMENT}

The authors would like to acknowledge the support of the National Science Foundation through the Materials Research Science and Engineering Center for Nanostructured Materials and Interfaces (Grant No. DMR-9632527)

${ }^{1}$ J. Maynard, Phys. Today 49, 26 (1996).

${ }^{2}$ A. Migliori and J. L. Sarrao, Resonant Ultrasound Spectroscopy (Wiley, New York, 1997).

${ }^{3}$ R. G. Leisure and F. A. Willis, J. Phys.: Condens. Matter 9, 6001 (1997).
${ }^{4}$ A. Migliori and T. W. Darling, Ultrasonics S34, 473 (1996).

${ }^{5}$ A. Murray and J. M. M. Neilson, Med. Biol. Eng. 13, 19 (1975).

${ }^{6}$ A. Murray and J. M. M. Neilson, Med. Biol. Eng. 13, 29 (1975).

${ }^{7}$ L. Auenbrugger, Inventum Novum ex Percussione Thoracis Humani ut Signo Obstrusos Interni Pectoris Morbos Detegendi, translated by Forbes, 1824 (Dawsons, London, Reprinted 1966).

${ }^{8}$ R. K. Lippmann, J. Bone Jt. Surg. 14, 118 (1932).

${ }^{9}$ V. T. Kuokkala and R. B. Schwarz, Rev. Sci. Instrum. 63, 3136 (1992).

${ }^{10}$ Y. Sumino, I. Ohno, T. Goto, and M. Kumazawa, J. Phys. Earth 24, 263 (1976).

${ }^{11}$ R. S. Lakes and J. Quackenbush, Philos. Mag. Lett. 74, 227 (1996).

${ }^{12}$ R. D. Mindlin, J. Appl. Phys. 27, 1462 (1956).

${ }^{13}$ I. S. Sokolnikoff, Mathematical Theory of Elasticity (Krieger, New York, 1983).

${ }^{14}$ R. D. Demarest, Jr., J. Acoust. Soc. Am. 49, 768 (1971).

${ }^{15}$ R. D. Mindlin, Int. J. Solids Struct. 12, 27 (1975).

${ }^{16}$ W. G. Gottenberg and R. M. Christensen, Int. J. Eng. Sci. 2, 45 (1964).

${ }^{17}$ E. J. Graesser and C. R. Wong, Mechanics and Mechanisms of Material Damping, edited by V. K. Kinra and A. Wolfenden (ASTM, Philadelphia, PA, 1992).

${ }^{18}$ C. P. Chen and R. S. Lakes, J. Rheol. 33, 1231 (1989).

${ }^{19}$ R. S. Lakes, Viscoelastic Solids (Chemical Rubber Corp., Boca Raton, FL, 1999).

${ }^{20}$ V. J. Koppellmann, Rheol. Acta 1, 20 (1958).

${ }^{21}$ W. Duffy, J. Appl. Phys. 68, 5601 (1990).

${ }^{22}$ M. Brodt, L. S. Cook, and R. S. Lakes, Rev. Sci. Instrum. 66, 5292 (1995).

${ }^{23}$ V. B. Braginskii, V. P. Mitrofanov, and V. I. Panov, Systems with Small Dissipation (University of Chicago Press, Chicago, 1985).

${ }^{24}$ H. Zhang et al., J. Acoust. Soc. Am. 103, 2385 (1998). 